\title{
Study of rare decays at CMS
}

\author{
Dayong Wang ${ }^{* \dagger}$ \\ for the CMS Collaboration
}

State Key Laboratory of Nuclear Physics and Technology, Peking University, Beijing, China

E-mail: dayong.wang@pku. edu.cn

Rare decays of heavy flavors and tau leptons could be very sensitive probes for searching for new physics. This presentation summarizes some recent CMS results on rare decays with different data sets. The angular analyses of rare $b \rightarrow s \ell^{+} \ell^{-}$transition processes of $\mathrm{B}^{0} \rightarrow \mathrm{K}^{* 0} \mu^{+} \mu^{-}$ and $\mathrm{B}^{+} \rightarrow \mathrm{K}^{+} \mu^{+} \mu^{-}$show consistent values of the angular distribution parameters with SM predictions. The $\mathrm{B}_{s}^{0} \rightarrow \mu^{+} \mu^{-}$decay is observed with a significance of 5.6 standard deviations and the time-integrated branching fraction is measured as $\mathscr{B}\left(\mathrm{B}_{\mathrm{s}}^{0} \rightarrow \mu^{+} \mu^{-}\right)=\left[2.9_{-0.6}^{+0.7}(\exp ) \pm\right.$ 0.2 (frag) $] \times 10^{-9}$, with effective lifetime $\tau_{\mu^{+} \mu^{-}}=1.70_{-0.44}^{+0.61} \mathrm{ps}$. The search for $\mathrm{B}^{0} \rightarrow \mu^{+} \mu^{-}$constrains the branching fraction to be $<3.6 \times 10^{-10}$ at $95 \%$ confidence level. The search for $\tau \rightarrow 3 \mu$ decays sets an upper limit of $\mathscr{B}(\tau \rightarrow 3 \mu)<8.8 \times 10^{-8}$ at $90 \%$ confidence level. All these results are consistent with the Standard Model predictions. Efforts with more channels and more coming data will be continued to further test the Standard Model with higher precision in future. Some prospects towards HL-LHC are also discussed.

XXIX International Symposium on Lepton Photon Interactions at High Energies - LeptonPhoton2019 August 5-10, 2019

Toronto, Canada

\footnotetext{
* Speaker.

${ }^{\dagger}$ Partially supported by the Ministry of Science and Technology of China, under Grant No. 2018YFA0403900 and National Science Foundation of China under Grant No.11661141008.
} 


\section{Introduction}

Phenomena beyond the Standard Model (SM) of particle physics can become manifest directly, via the production of new particles, or indirectly, by affecting the production and decay of SM particles. Rare decays of heavy flavors and tau leptons could be very sensitive to these processes and ideal for probing the new physics. The CMS [1] at CERN is a general-purpose detector at the Large Hadron Collider (LHC). It is equipped with large area of silicon trackers, a 3.8T magnetic field, superb muon detection systems with large acceptance and very flexible trigger systems. These features make CMS an ideal for studying the rare decays of heavy flavors and tau leptons.

\section{Angular analyses of $b \rightarrow s \mu^{+} \mu^{-}: \mathrm{B}^{0} \rightarrow \mathrm{K}^{* 0} \mu^{+} \mu^{-}$[2] and $\mathrm{B}^{+} \rightarrow \mathrm{K}^{+} \mu^{+} \mu^{-}$[3]}

The transitions of the type $b \rightarrow s \ell^{+} \ell^{-}$is a flavor-changing neutral current (FCNC) process, with $\ell$ denoting a charged lepton. In the SM, this type of transition is forbidden at tree level and occurs through higher-order processes. New physics may modify any of the angular variables relative to their SM values, which makes these rare FCNC decays sensitive to possible physics phenomena beyond the SM. CMS has recently analysed two such FCNC decays: $\mathrm{B}^{0} \rightarrow \mathrm{K}^{* 0} \mu^{+} \mu^{-}$ and $\mathrm{B}^{+} \rightarrow \mathrm{K}^{+} \mu^{+} \mu^{-}$. Both analyses use a sample of events collected in proton-proton collisions at $8 \mathrm{TeV}$. The data correspond to an integrated luminosity of $20.5 \mathrm{fb}^{-1}$. The data for these analysis was recorded using a low-mass dimuon High Level Trigger with a displaced vertex.

The differential decay rate for $\mathrm{B}^{0} \rightarrow \mathrm{K}^{* 0} \mu^{+} \mu^{-}$can be written in terms of the dimuon mass squared $\left(q^{2}\right)$ and three angular variables $\left(\theta_{\ell}, \theta_{K}\right.$ and $\left.\varphi\right)$ as a combination of spherical harmonics. Measurements of the most angular variables by different experiments are consistent with the SM, except $P_{5}^{\prime}$ from a LHCb measurement [4] showed a potential discrepancy, thus of particular interest. CMS performed a new measurement of the $P_{1}$ and $P_{5}^{\prime}$ parameters [2] to elucidate the situation.
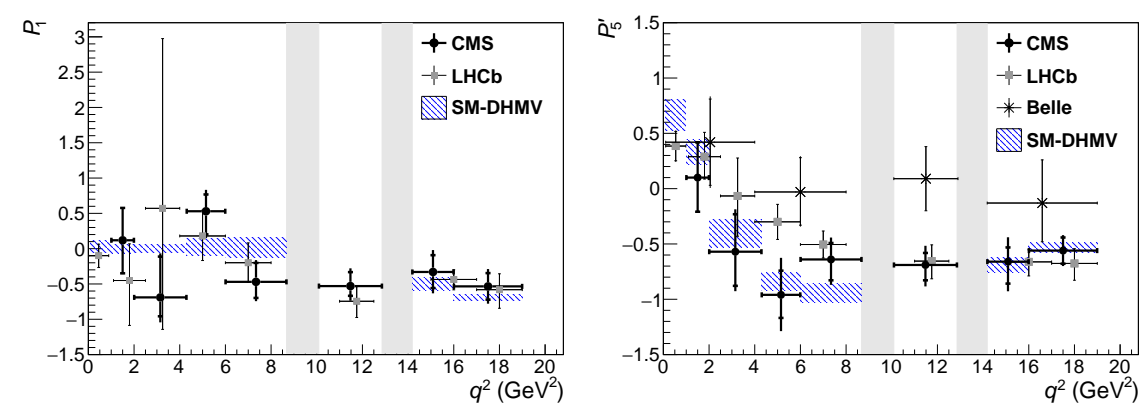

Figure 1: CMS measurements of the (left) $P_{1}$ and (right) $P_{5}^{\prime}$ angular parameters versus $q^{2}$ for $\mathrm{B}^{0} \rightarrow \mathrm{K}^{* 0} \mu^{+} \mu^{-}$decays, in comparison to results from the LHCb, Belle, and SM prediction (denoted as DHMV after the authors). The vertical shaded regions correspond to the $J / \psi$ and $\psi^{\prime}$ resonances.

In the measurement, the values of $P_{1}$ and $P_{5}^{\prime}$ are determined by fitting the distribution of events as a function of the three angular variables. All measurements are performed in $q^{2}$ bins from 1 to $19 \mathrm{GeV}^{2}$. The $q^{2}$ bins corresponding to $\mathrm{B}^{0} \rightarrow \mathrm{K}^{* 0} J / \psi$ and $\mathrm{B}^{0} \rightarrow \mathrm{K}^{* 0} \psi^{\prime}$ decays are used to validate the analysis. The fitted values of $P_{1}$, and $P_{5}^{\prime}$, along with their associated uncertainties, for each of 
the $q^{2}$ regions are shown in Fig. 1, along with the SM prediction. The results are among the most precise to date for these parameters and are consistent with predictions based on the SM.

Similarly, the $\mathrm{B}^{+} \rightarrow \mathrm{K}^{+} \mu^{+} \mu^{-}$decay rate depends on the angle between the directions of the $\mu^{-}$and $\mathrm{K}^{+}$in the dilepton rest frame $\cos \theta_{\ell}$, parametrized in terms of the forward-backward asymmetry $A_{\mathrm{FB}}$ of the dimuon system and the contribution $F_{\mathrm{H}}$ from the pseudoscalar, scalar, and tensor amplitudes to the decay width. In the analysis, $A_{\mathrm{FB}}$ and $F_{\mathrm{H}}$ are extracted from a two-dimensional extended unbinned maximum-likelihood fit to the angular distribution of the selected $\mathrm{B}^{+}$meson candidates in each $q^{2}$ range from 1 to $22 \mathrm{GeV}^{2}$. The measured values of $A_{\mathrm{FB}}$ and $F_{\mathrm{H}}$ for each $q^{2}$ range are shown in Fig. 2. The results of $A_{\mathrm{FB}}$ are consistent with the SM expectation of no asymmetry and $F_{\mathrm{H}}$ also consistent with the SM calculation. There is generally good agreement between the predictions and our results, as well as between our results and previous measurements.
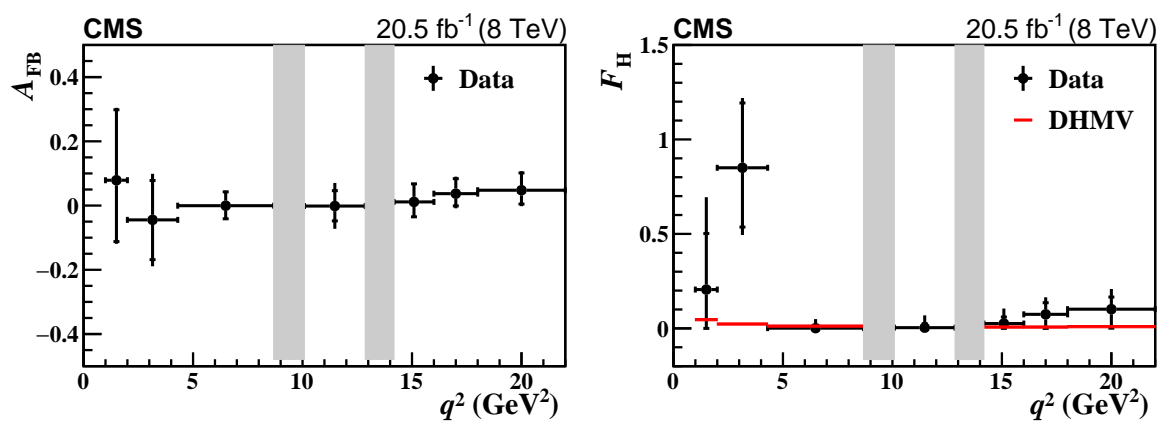

Figure 2: Results of $A_{\mathrm{FB}}$ (left) and $F_{\mathrm{H}}$ (right) in bins of $q^{2}$. The red line shows the SM prediction.

With increased statistics at Run-II, a full angular analysis without simplification is possible for $\mathrm{B}^{0} \rightarrow \mathrm{K}^{* 0} \mu^{+} \mu^{-}$. Also possible are angular analyses of more b-hadron channels of $\mathrm{B}^{0}, \mathrm{~B}^{+}, \mathrm{B}_{\mathrm{s}}{ }^{0}$, $\Lambda_{\mathrm{b}}{ }^{0}$ etc. At HL-LHC, with the large data set of $3000 \mathrm{fb}^{-1}$, the $P_{5}^{\prime}$ uncertainties in the $q^{2}$ bins are estimated to improve by up to a factor of 15 compared to the measurement of $8 \mathrm{TeV}$ [5].

\section{Measurement of properties of $\mathrm{B}_{\mathrm{s}}^{0} \rightarrow \mu^{+} \mu^{-}$decays and search for $\mathrm{B}^{0} \rightarrow \mu^{+} \mu^{-}$[6]}

Measurements of the rare leptonic $\mathrm{B}$ meson decays $\mathrm{B}_{\mathrm{s}}^{0} \rightarrow \mu^{+} \mu^{-}$and $\mathrm{B}^{0} \rightarrow \mu^{+} \mu^{-}$are performed in a combined data sample of $5 \mathrm{fb}^{-1}$ at $7 \mathrm{TeV}, 20 \mathrm{fb}^{-1}$ at $8 \mathrm{TeV}$, and $36 \mathrm{fb}^{-1}$ at $13 \mathrm{TeV}$. The dimuon invariant mass with the fit projection and the likelihood contours are shown in Fig. 3.

The $\mathrm{B}_{\mathrm{s}}^{0} \rightarrow \mu^{+} \mu^{-}$decay is observed with a significance of 5.6 standard deviations and the time-integrated branching fraction is measured as $\mathscr{B}\left(\mathrm{B}_{\mathrm{s}}^{0} \rightarrow \mu^{+} \mu^{-}\right)=\left[2.9_{-0.6}^{+0.7}(\exp ) \pm 0.2(\right.$ frag $\left.)\right] \times$ $10^{-9}$, where the experimental uncertainty combines the statistical and systematic terms. The second uncertainty refers to the uncertainty in the ratio $f_{s} / f_{u}$ of the $\mathrm{B}_{\mathrm{s}}^{0}$ and the $\mathrm{B}^{+}$fragmentation functions. No significant $\mathrm{B}^{0} \rightarrow \mu^{+} \mu^{-}$signal is observed and an upper limit $\mathscr{B}\left(\mathrm{B}^{0} \rightarrow \mu^{+} \mu^{-}\right)<3.6 \times 10^{-10}$ is determined at $95 \%$ confidence level. The $\mathrm{B}_{\mathrm{s}}^{0} \rightarrow \mu^{+} \mu^{-}$effective lifetime, measured for the first time by CMS, is found to be $\tau_{\mu^{+} \mu^{-}}=1.70_{-0.44}^{+0.61} \mathrm{ps}$, where the uncertainty combines both statistical and systematic components. The results for the branching fractions supersede the previous CMS results based on the 7 and $8 \mathrm{TeV}$ data only. All results are in agreement with the SM predictions. 

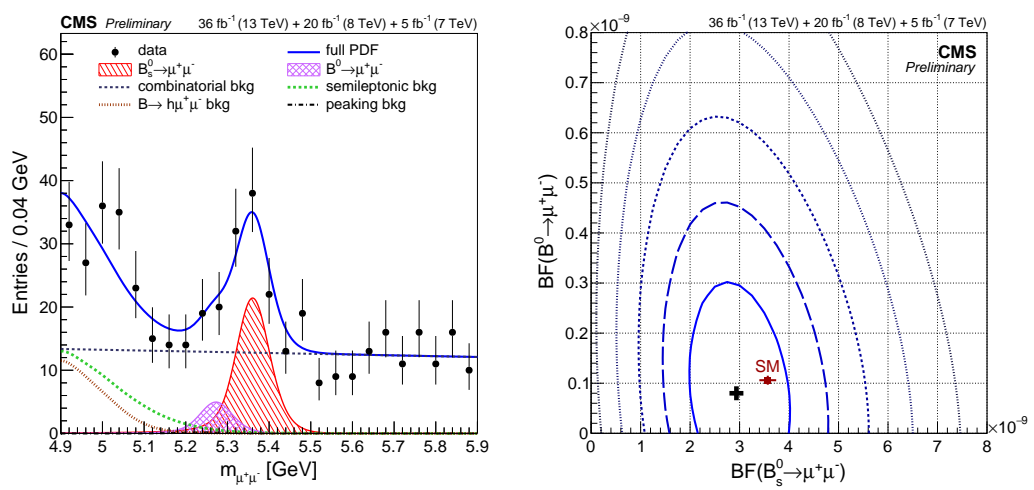

Figure 3: (Left) Invariant mass of dimuon distributions with the fit projection. (Right) Likelihood contours of 1-5 $\sigma$ coverage for the fit to $\mathscr{B}\left(\mathrm{B}_{\mathrm{s}}^{0} \rightarrow \mu^{+} \mu^{-}\right)$and $\mathscr{B}\left(\mathrm{B}^{0} \rightarrow \mu^{+} \mu^{-}\right)$, together with the SM expectation.

At future HL-LHC, the upgraded detector with improved momentum resolution, is expected to enable high precision measurements of the branching fractions and the effective lifetime of $\mathrm{B}_{\mathrm{s}}^{0} \rightarrow \mu^{+} \mu^{-}$with reduced systematic and statistical uncertainties. At $3000 \mathrm{fb}^{-1}$, it will be possible to observe the $\mathrm{B}^{0} \rightarrow \mu^{+} \mu^{-}$decay with over $5 \sigma$ significance [7].

\section{Search for $\tau \rightarrow 3 \mu$ decays [8]}

In the SM, there are no known symmetries that strictly forbid charged lepton flavor violating (CLFV) decays, such as $\ell \rightarrow \ell^{\prime} \gamma, \ell \rightarrow 3 \ell^{\prime}$. Due to neutrino oscillations, such decays are possible, albeit with extraordinarily small branching fractions. SM extensions often lead to CLFV decays with appreciable branching fractions that can be well within the current experimental sensitivity.

The first search for charged lepton flavor violating decays $\tau \rightarrow 3 \mu$, using CMS detector is performed. The search uses $33 \mathrm{fb}^{-1}$ of proton-proton collisions collected at $13 \mathrm{TeV}$ by CMS in 2016. It exploits $\tau$ leptons produced in $\mathrm{D}$ and $\mathrm{B}$ meson decays, which is the main source of $\tau$ leptons at the LHC. No excess above the expected background is observed. An upper limit of $8.8 \times 10^{-8}$ is set on the branching fraction $\mathscr{B}(\tau \rightarrow 3 \mu)$ at $90 \%$ confidence level. The corresponding upper limit at $95 \%$ confidence level is $1.1 \times 10^{-7}$.

\section{References}

[1] CMS Collaboration, S. Chatrchyan et al., JINST 3, S08004 (2008).

[2] CMS Collaboration, A. M. Sirunyan et al., Phys. Lett. B781, 517 (2018).

[3] CMS Collaboration, A. M. Sirunyan et al., Phys. Rev. D 98, 112011 (2018).

[4] LHCb Collaboration, R. Aaij et al., Phys. Rev. Lett. 111, 191801 (2013).

[5] CMS Collaboration, Report No. CMS-PAS-FTR-18-033, Dec. 2018.

[6] CMS Collaboration, Report No. CMS-PAS-BPH-16-004, Aug. 2019.

[7] CMS Collaboration, Report No. CMS-PAS-FTR-18-013, Dec. 2018.

[8] CMS Collaboration, Report No. CMS-PAS-BPH-17-004, Mar. 2019. 